it speaks a language the student can easily understand. Numerous examples and problems are given with every topic. We welcome Chapter XIV on computation, approximations, differences, and interpolation. The requirements of practical computation are responsible for so many of the algebraic reductions with which our classes struggle, that a first hand experience with the problems of computation must help the students to see the reasons for some of the hardships inflicted upon them. The review in Chapters I and II of those parts of the high school work which are stumbling blocks for most freshmen and the discussion of division by 0 and of infinity will be appreciated by those who teach freshmen classes.

The definition of infinite series in $\$ 196$ confuses the notion of series with that of sequence. In $\S 246$ we read " An arrangement of the numbers $1,2,3, \ldots, n$ is called an inversion," a statement which attributes to this word a meaning not usually given it.

Many of the subjects taken up require a more rigorous treatment than it would be wise to give them in an introductory text. In most such cases the finer points are not slurred over, but clearly indicated and then taken for granted, as, for instance, in discussing $\lim _{z=\infty}(1+1 / z)^{z}$, in deriving Maclaurin's series, etc.

The last sixty pages of the book contain the answers for the odd-numbered exercises, an index, and two appendices. The first of the appendices gives a list of formulas, definitions, and theorems. The second includes, besides the tables commonly found, also a conversion table for changing from sexagesimal to radian measure, and conversely; one for $\log _{e} x, e^{x}$, and $e^{-x}$ from $x=0$ to $x=5$ at intervals of 0.05 ; and one for the squares and cubes, square and cube roots of numbers between 1 and 100. A handy cardboard protractor-ruler accompanies the book.

Among the misprints, we notice $c$ in place of $c^{2}$ on page 23, $2 \mathrm{~d}$ line from the top; and $\sqrt[4]{9.35}$ in place of $\sqrt[4]{9.35^{3}}$ on page 29 , 10th line from the bottom.

Arnold Dresden.

College Algebra. By Schuyler C. Davisson, Рh.D. New York, The Macmillan Co., 1910. Small 8vo. xvi +243 pp. $\$ 1.50$ net.

IN this book no attempt at correlation is made; it is a pres- 
entation of the topics usually taken up in the freshman class in algebra, different in many respects however from the usual text.

The preface states that "the book is not written for mathematicians, but for students desiring to know the elements of ordinary algebra." Can this be said to describe the state of mind of the majority of our college freshmen? Had we not better face our problem squarely and recognize the situation we have to deal with in our classes? As it appears to the reviewer, of those entering the freshman class in mathematics, not many have a desire to know the elements of ordinary algebra. At best, their mind is void of any desire with regard to algebra ; if we can succeed in arousing their interest, we may be able to call it forth ; but, to count on it at the beginning of the college course, seems too optimistic. It is for this reason that the book does not seem suitable for the ordinary freshman course, to which students are admitted who have had one and a half years of secondary algebra.

That the author seems to have had in mind students who have had more than one and a half years of preparatory work, or at least students who have to some extent found themselves with respect to algebra, would also appear from the following paragraph in the preface: "The text does not contain a very large number of problems, but it is believed to contain a sufficient number to illustrate the theory. The aim has been to give the student an idea that algebra consists of something more than problem solving." Assuming this to be the author's point of view, the present writer believes that we have here an excellent text before us.

Part I-entitled Numbers and written, as is Chapter XV on Limits, by Professor A. M. Kenyon - opens with a presentation of the system of natural numbers and the laws governing the operations upon them. In order to make the inverse operations of division, evolution, and subtraction possible within the domain, successive extensions of the number system are made, obtaining step by step the domains of positive rational numbers, positive real numbers, real numbers, and complex numbers. Few theorems are proved in this chapter. It seems that the presentation is somewhat difficult to follow for one who is making his first acquaintance with the subject. Greater unity might have been obtained if, the positive rational numbers having been introduced as pairs $(a, b)$ of integers whose order relation is 
defined by

$$
(a, b) \lesseqgtr(c, d) \text { according as } a d \lesseqgtr b c,
$$

the negative rational numbers had been introduced as pairs $(a, b)$ of integers whose order relation is given by

$$
(a, b) \lesseqgtr(c, d) \text { according as } a+d \lesseqgtr b+c .
$$

The chapter would also have gained strength if the theorems concerning at least one of the domains considered had been stated and proved in terms of the original definition of the numbers of the domain as number pairs.

In $\S 40$, the student is asked to show that the definition of addition for rational numbers is consistent with the one given for the natural numbers ; similar exercises are set for the other operations and extensions. Would it not be well to emphasize more strongly the important point involved?

Part II - Algebra - opens with Chapter II on definitions, followed by Chapter III on fundamental laws and identities. The definition of an algebraic function in $\S 132$ as "an algebraic combination of quantitative and operative symbols that has meaning" seems unsatisfactory. What is an "algebraic combination of symbols"? How can we tell whether such a combination has meaning, unless we know in what domain we are working? Would $2^{2}-3+5^{3}$ not be an algebraic function under this definition? Have we not here a confusion of the notions function and functional value? About variables and constants, $\S 133$ says, among other things: "When several literal quantities are involved in a function, the first letters of the alphabet shall be considered as constants, while the last letters shall be considered as variables," a statement which does not give any information whatever about the concept variable.

The remaining chapters, on factorization of rational integral functions; equations in one and more unknowns and determinants; indices, surds, and complex numbers; sequences; permutations and combinations; mathematical induction; undetermined coefficients and partial fractions; linits and infinite series; theory of equations; logarithms and variation, contain a very attractive treatment of these various topics, presenting several features with regard to subject matter as well as arrangement that are different from what is usual in a college algebra. Mention should be made of the following points : 
The section on factorization of symmetric functions in Chapter IV ; the determination of the integral roots of equations of higher degree than the second in Chapter $\mathrm{V}$, rather than in the later chapter on the theory of equations; the treatment of determinants in the chapter on linear simultaneous equations and the application of determinants to the solution of systems of $n$ linear homogeneous equations in $n$ variables and of $n$ linear non-homogeneous equations in $n$ or in $n-1$ variables.

The treatment of the theory of limits in Chapter XV should be noticed for its excellence; the substance of the $\epsilon, \delta$ definitions of limits is given verbally, and illustrated graphically without using the algebraic statement of the definitions; the presentation of infinite sequences and series becomes clear as a result of this treatment of limits.

The exercises in verbally expressing the formulas used are a welcome aid in the efforts to combat the carelessness of expression to which our students are so largely accustomed. The method of graphing a function is explained in Chapter V, but is made very little use of in the later chapters. One misses the graphical illustrations particularly in the chapters on simultaneous equations and in the chapter on variation, while the graphical methods could have been used to greater advantage in the chapter on theory of equations and in the chapter on limits.

\section{Arnold Dresden.}

Spezielle algebraische und tranzendente ebene Kurven, Theorie und Geschichte. Von Gino Loria. Autorisierte nach dem italienischen Manuskript bearbeitete Ausgabe von F. SchüTTE. Zweite Auflage. Leipzig, B. G. Teubner. Erster Band: Die algebraischen Kurven, 488 pp. and 14 plates, 1910. Zweiter Band: Die tranzendenten und abgeleiteten Kurven, 384 pp. and 6 plates, 1911.

THE value and importance of this work, which was first published in 1902, are partially indicated by this early appearance of a complete revision. About one hundred and twenty-five pages and three plates have been added. Since 1902 much has been done in the theory of special plane curves, and this work has, as far as possible, been incorporated in the new edition, which is now published in two volumes instead of one.

Many of the excellent features of the work were pointed out and discussed at some length in Professor E. B. Wilson's 